\section{Time for a change and to adopt a novel molecular genomic approach in NETs}

\author{
Lisa Bodei, Eric Liu, Scott Paulson, Anthony Gulati, Jon Freudman, \\ William Grosh, Sheldon Kafer, Prasanna C. Wickremesinghe and \\ Ronald R. Salem
}

We read with interest the News and Views article by Rindi and Wiedenmann (Neuroendocrine neoplasia goes molecular - time for a change. Nat. Rev. Clin. Oncol. 16, 149-150 $(2018))^{1}$, two notable and eminent authorities in the field of genomic medicine. Despite the concerns raised by these experts, the registry study discussed in their article unequivocally demonstrated the clinical utility of the NETest liquid biopsy assay in the 'real-world' setting ${ }^{2}$. NETest scores enabled accurate prediction $(100 \%)$ of disease progression and had a $98 \%$ correlation with response to therapy, reducing the requirement to perform an imaging test in $40 \%$ of patients.

NETest is the first blood-based multianalyte gene transcript (mRNA-based) test for neuroendocrine tumours (NETs). The genomic validity of this assay was independently confirmed in an NIH-funded study evaluating the gene expression profiles of $>10,000$ tumours from The Cancer Genome Atlas (TCGA) database, with the results indicating that the NETest gene signature accurately captured the NET phenotype ${ }^{2}$. Our registry study confirms the clinical utility of this signature.

The following factual inaccuracies were stated in the News and Views by Rindi and Wiedenmann ${ }^{1}$ : that the Response Evaluation Criteria in Solid Tumors (RECIST) always enable an accurate assessment of NET progression; that our data should be viewed cautiously owing to tumour heterogeneity; that we provided no sensitivity data; that patient management depends on a multidisciplinary team (MDT) approach; and that the cost-benefit ratio of NETest is unfavourable.

First, RECIST criteria are of limited value in the assessment of indolent tumours, such as NETs, and have no predictive value in this disease ${ }^{3}$. The RECIST are a 'trial tool' not used in clinical practice. NETest, however, has clinical utility as the first blood-based objective genomic assay to facilitate identification of the molecular mechanisms that define individual tumour behaviour. NETest enables detection of $1 \mathrm{cell} / \mathrm{ml}$ of blood and is $\sim 30,000-50,000$ times more sensitive than imaging, which provides only volumetric data ${ }^{4}$.

Second, the real-world study captured the usual mix of NETs commonly encountered by physicians. We agree that tumour heterogeneity is an issue when performing tissue biopsies; hence, the limited value of NET tissue biopsy analysis as an accurate prognostic tool ${ }^{5}$. Using liquid biopsy approaches, however, all clonal components are sampled. NETest was effective as a diagnostic and prognostic tool in grade 1-2 tumours ${ }^{4}$, making it evident that heterogeneity is an issue derived from performing tissue biopsy.

Third, our clinical study was not designed to examine laboratory parameters because such data has already been extensively published. For example, the diagnostic accuracy of NETest for either lung or gastroenteropatic NETs is $>90 \%{ }^{4}$ - in the registry study ${ }^{2}$, it was $96 \%$ irrespective of tumour origin, with a coefficient of variation $(\mathrm{CV})$ of $<5 \%$. Both the diagnostic and the laboratory analytic metrics exceeded those obtained using serum chromogranin A (CgA) assays. The latter biomarker exhibits a diagnostic accuracy of $\sim 50-70 \%$ and a CV of $<20 \%$. NETest is specifically designed to be used for patient management and was $85 \%$ concordant with the disease status (for example, stable or progressive). This percentage surpasses the $80 \%$ metrics proposed by the $\mathrm{NIH}^{6}$ and exceeds the performance of $\mathrm{CgA}$ assays, which have $\mathrm{a}<50 \%$ concordance with disease status ${ }^{7}$.

Fourth, an MDT approach is important in the management of patients with all tumour types and not just those with NETs. The key issue with MDTs is that their validity in making consensus decisions is only as effective as the information they consider. MDTs evaluate imaging data, which can be interpreted subjectively and can be unreliable in guiding the management of patients with NETs ${ }^{8}$. In our study, a low NETest score enabled the identification of patients with molecularly stable disease. As a consequence,
$40 \%$ of patients required fewer follow-up interventions than they would have received if they had been evaluated according to standard procedures. This result has obvious clinical and economic implications.

Fifth, blood biomarker information can provide an indication of disease status earlier than is possible with imaging and thereby facilitate earlier cessation of an ineffective therapy - an obvious cost benefit ${ }^{9}$. Economic modelling studies conclude that patient monitoring with NETest would substantially decrease the expenditure in dollars per year per patient. Imaging costs are US\$6,000-10,000 per event and the cost of standard-of-care treatments (such as somatostatin analogues, peptide receptor radionuclide therapy or everolimus) are $\$ 50,000-100,000$ per year per patient. Thus, a biomarker that enables decreased use of interventions in $40 \%$ of patients and alters the management of another $40 \%$ has cogent economic implications.

Iconoclastic views of novel sophisticated technologies has culminated in the management of NETs falling behind other oncological disciplines (such as the management of breast cancer $)^{10}$, which ultimately is detrimental to patients. Rindi and Wiedenmann disappointingly reiterate the tautology "reaching perfection is almost impossible in the real world" ${ }^{\text {. On }}$ the contrary, only through the thoughtful adoption of novel advances rather than clinging to dogma can medicine reach for perfection and thereby provide the best care for our patients ${ }^{4}$.

There is a reply to this correspondence by Rindi, G. \& Wiedenmann, B. Nat. Rev. Clin. Oncol. https://doi.org/10.1038/s41571-0190186-4 (2019).

\section{Lisa Bodei ${ }^{*}$, Eric Liu'2, Scott Paulson ${ }^{3}$, Anthony Gulati Jon Freudman ${ }^{5}$. William Grosh ${ }^{6}$, Sheldon Kafer Prasanna C. Wickremesinghe ${ }^{8}$ and Ronald R. Salem ${ }^{9}$ \\ 'Department of Radiology, Memorial Sloan Kettering Cancer Center, New York, NY, USA ${ }^{2}$ Rocky Mountain Cancer Center, Denver, CO, USA \\ ${ }^{3}$ Texas Oncology-Baylor Charles A Sammons Cancer Center, Dallas, TX, USA. "Bennett Cancer Center, Stamford, CT, USA. ${ }^{5}$ Freudman Healthcare Consulting, San Rafael, CA, USA. \\ ${ }^{6}$ Emily Couric Cancer Center, University of Virginia, Charlottesville, VA, USA \\ ${ }^{7}$ Department of Internal Medicine, Hartford Hospital, Hartford, CT, USA \\ ${ }^{8}$ Department of Gastroenterology, Richmond University Medical Center, Staten Island, NY, USA ${ }^{9}$ Department of Surgery, Yale University School of Medicine, New Haven, CT, USA \\ *e-mail: bodeil@mskcc.org https://doi.org/1 0.1038/s41571-019-0185-5}


1. Rindi, G. \& Wiedenmann, B. Neuroendocrine neoplasia goes molecular - time for a change. Nat. Rev. Clin. Oncol. 16, 149-150 (2018)

2. Liu, E. et al. Assessment of NETest clinical utility in a US registry-based study. Oncologist https://doi.org/10.1634/theoncologist.2017-0623 (2018).

3. de Mestier, L. et al. Evaluating digestive neuroendocrine tumor progression and therapeutic responses in the era of targeted therapies: state of the art. Endocr. Relat. Cancer. 21, R105-R120 (2014).

4. Modlin, I. M. et al. The NETest: the clinical utility of multigene blood analysis in the diagnosis and management of neuroendocrine tumors. Endocrinol. Metab. Clin. North Am. 47, 485-504 (2018).

Tang, L. H. et al. Objective quantification of the Ki67 proliferative index in neuroendocrine tumors of the gastroenteropancreatic system: a comparison of digital image analysis with manual methods. Am. J. Surg. Pathol. 36, 1761-1770 (2012).
6. Bossuyt, P. M. et al. The STARD statement for reporting studies of diagnostic accuracy: explanation and elaboration. Ann. Intern. Med. 138, W1-W12 (2003).

7. Marotta, V. et al. Chromogranin A as circulating marker for diagnosis and management of neuroendocrine neoplasms: more flaws than fame. Endocr. Relat. Cancer. 25, R11-R29 (2018).

8. Capdevila, J. et al. Translational research in neuroendocrine tumors: pitfalls and opportunities. Oncogene 36, 1899-1907 (2017).

9. Strosberg, J. et al. United States-based practice patterns and resource utilization in advanced neuroendocrine tumor treatment. World J. Gastroenterol. 19, 2348-2354 (2013).

10. Sparano, J. A. et al. Adjuvant chemotherapy guided by a 21 -gene expression assay in breast cancer. N. Engl. J. Med. 379, 111-121 (2018)

Competing interests

The authors declare no competing interests.

\section{Reply to 'Time for a change and to adopt a novel molecular genomic approach in NETs'}

\section{Guido Rindi(D) and Bertram Wiedenmann}

We wish to respond to the letter from Lisa Bodei and collaborators (Bodei, L. et al. Time for a change and to adopt a novel molecular genomic approach in NETs. Nat. Rev. Clin. Oncol. https://doi.org/10.1038/s41571-0190185-5 (2019) $)^{1}$ in which they comment on our News \& Views piece (Rindi, G. \& Wiedenmann, B. Neuroendocrine neoplasia goes molecular - time for a change. Nat. Rev. Clin. Oncol. 16, 149-150 (2018)) $)^{2}$ on their article describing the prospective evaluation of the NETest in a cohort of 100 patients ${ }^{3}$. In their letter, Bodei et al. quote “.... an NIHfunded study evaluating the gene expression profiles of $>10,000$ tumours from The Cancer Genome Atlas (TCGA) database..."1 and cite their study ${ }^{3}$ to support this statement, but they ultimately fail to provide the claimed data. We wish to highlight that we were asked to scrutinize and comment on the data provided by Liu et al. ${ }^{3}$ and not the entire set of data generated by the use of the NETest. We feel that this premise is indicative of how Bodei and collaborators interpreted our commentary.

In their letter, Bodei et al. list five "factual inaccuracies" ${ }^{\prime 1}$ of our News \& Views article that we wish to clarify. First, according to the correspondents, we claimed that the Response Evaluation Criteria in Solid Tumors (RECIST) always enable an accurate assessment of the progression of neuroendocrine tumours (NETs). No such statement was made ${ }^{2}$, and neither were we asked to express our opinion on the efficacy of RECIST in clinical practice. However, it is factually accurate that they used RECIST in their study ${ }^{3}$ to evaluate tumour progression and compare with the results obtained with the NETest.

Second, Bodei et al. seem to imply that we recommended their data should be viewed with caution owing to intra-patient tumour heterogeneity. Given the high degree of heterogeneity of neuroendocrine neoplasia, however, we focused only on inter-patient heterogeneity and indeed we suggested that the application of this test would be challenged in larger patient cohorts stratified by site, tumour type, grade and/or stage ${ }^{2}$. Inter-patient tumour heterogeneity remains a crucial issue at different levels (clinical, genetic and circulating markers, including RNA and DNA). Blood-based tests do not necessarily reflect the same relative proportions of tumour-related RNA in the bloodstream as might be present in tumour cells owing to the occurrence of tumour cell anoikis or differentiation and to differences in cell volume. Their statement "Using liquid biopsy approaches, however, all clonal components are sampled" ${ }^{1}$ is currently unrealistic . $^{4}$ Furthermore, no evidence is available to indicate whether blood and tissue samples are subject to the same sampling limitations - indeed, cancer is a dynamic entity in which the levels of both RNA transcripts and protein expression change over time. Finally, the NETest does not encompass all the possible alterations in NETs, thus limiting its potential for general application ${ }^{3}$.

The third factual inaccuracy that Bodei et al. found in our News \& Views was the statement that they did not provide sensitivity data in their study ${ }^{3}$. Indeed, the data presented in the study do not enable robust calculations of the sensitivity of the NETest, including negative and positive predictive values, and none of these data was openly displayed.

Fourth, they comment that our statement that patient management depends on a multidisciplinary team (MDT) approach should be applied to all cancer types and not only to patients with NETs. We agree and insist that MDTs should continue to be considered as a mainstay of the management of these patients.

Finally, Bodei and collaborators attribute to us the statement that the cost-benefit ratio of the NETest is unfavourable. No such statement is present in our News \& Views ${ }^{2}$; rather, we expressed our general opinion about all blood-based molecular tests. Regardless, in the European setting of universal health-care coverage, the cost of such assays does, unfortunately, remain an issue.

In our opinion, the critical interpretation by Bodei et al. of our commentary is factually misplaced. Unfortunately, and for reasons we do not understand, Bodei et al. failed to appreciate the overall positive vision we provided about molecular testing in neuroendocrine cancer, both upfront in the title and in the good-news statement conclusion of our commentary ${ }^{2}$. We also certainly stressed the need for better work to understand the efficacy and limitations of such tests in clinical practice, but measurable advances will only be achieved when the dialogue between science and medicine is preserved.

Guido Rindi $\mathbb{D}^{1 *}$ and Bertram Wiedenmann ${ }^{2}$

'Fondazione Policlinico Universitario A. Gemelli IRCCS-Università Cattolica del Sacro Cuore, Rome, Italy.

${ }^{2}$ Charité, Campus Virchow Klinikum and Charité Mitte, University Medicine Berlin, Berlin, Germany. *e-mail: guido.rindi@unicatt.it https://doi.org/10.1038/s41571-019-0186-4

1. Bodei, L. et al. Time for a change and to adopt a novel molecular genomic approach in NETs. Nat. Rev. Clin. Oncol, https://doi.org/10.1038/s41571-019-0185-5 (2019).

2. Rindi, G. \& Wiedenmann, B. Neuroendocrine neoplasia goes molecular - time for a change. Nat. Rev. Clin. Oncol. 16, 149-150 (2018).

3. Liu, E. et al. Assessment of NETest Clinical utility in a U. S. registry-based study. Oncologist https://doi. org/10.1634/theoncologist.2017-0623 (2018). 4. Bardelli, A. \& Pantel, K. Liquid biopsies, what we do not know (yet). Cancer Cell 31, 172-179 (2017).

\section{Competing interests}

G.R. has received honoraria from Ipsen and Novartis for his role in their speaker's bureau. B.W. declares no competing interests. 